\title{
¿Son las Fuerzas Armadas actores humanitarios? La importancia del Estado y del ser humano a partir de la experiencia ecuatoriana de envío de tropas para misiones de reconstrucción en el Caribe*
}

María Augusta Pérez Aldaz ${ }^{a}$

Resumen: El presente artículo se enmarca en el área de relaciones internacionales, en la subárea de seguridad, pues aborda las actividades que realizan los militares en asuntos humanitarios, específicamente relacionados con desastres naturales. El argumento principal sostiene que los ejércitos, por su naturaleza, no son una institución humanitaria, debido a que su misión es la defensa y la seguridad de los Estados. Sin embargo, estos, basados en sus objetivos estratégicos, utilizan a sus ejércitos en asuntos humanitarios y reformulan sus intereses nacionales, gracias al paraguas de los derechos humanos y la importancia del ser humano frente al Estado. Para el efecto, los conceptos de seguridad y poder de la teoría realista son aplicados al caso ecuatoriano en la experiencia de las misiones de ayuda para la reconstrucción en el Caribe: Marhec, Marcec y Mahrgec.

Palabras clave: Estado; Fuerzas Armadas; intereses; poder; seguridad.

Fecha de recibido: 4/12/2018 Fecha de evaluación: 8/02/2019 Fecha de aprobado: 21/03/2019

* El presente artículo expone los resultados obtenidos en la tesis: "Las Fuerzas Armadas en asuntos humanitarios: el caso de Ecuador", de la maestría en investigación de Relaciones Internacionales con mención en Seguridad y Derechos Humanos de la Facultad Latinoamericana de Ciencias Sociales (Flacso), sede Ecuador.

a Abogada de la Pontificia Universidad Católica del Ecuador y estudiante de la Maestría de Relaciones Internacionales en la Facultad Latinoamericana de Ciencias Sociales (Flacso), sede Ecuador.

Correo electrónico: magusmariaperez_25@hotmail.com

ORCID: orcid.org/0000-0002-8944-3777 
Cómo citar: Pérez, M. A. (2019). ¿Son las Fuerzas Armadas actores humanitarios? La importancia del Estado y del ser humano a partir de la experiencia ecuatoriana de envío de tropas para misiones de reconstrucción en el Caribe. Revista Relaciones Internacionales, Estrategia Y Seguridad, 14(2). pp. 107131. https://doi.org/10.18359/ries.3805

\title{
Are Armed Forces Humanitarian Actors? The Importance of the State and the Human Being Based on the Ecuadorian Experience of Sending Troops to the Caribbean for Reconstruction Missions
}

\begin{abstract}
This article belongs to the field of international relations and the sub-field of security, since it deals with humanitarian operations carried out by the military, especially after natural disasters. The main argument is that, by nature, armies are not a humanitarian institution because their mission is to defend and secure states. However, based on their strategic objectives, states use their armies in humanitarian affairs and reformulate their national interests under the umbrella of human rights and the importance of the human being vis-à-vis the state. For this purpose, the concepts of security and power of the realistic theory are applied to the Ecuadorian case of reconstruction aid missions in the Caribbean: Marhec, Marcec and Mahrgec.
\end{abstract}

Keywords: State; Armed Forces; interests; power; security.

As Forças Armadas são atores humanitários? A importância do Estado e do ser humano a partir da experiência equatoriana de enviar tropas para missões de reconstrução no Caribe

\footnotetext{
Resumo: Este artigo se enquadra na área de relações internacionais, na subárea de segurança, pois aborda as atividades realizadas pelos militares em questões humanitárias, especificamente as relacionadas a desastres naturais. O principal argumento afirma que os exércitos, por sua natureza, não são uma instituição humanitária, porque sua missão é a defesa e segurança dos Estados. No entanto, os Estados, com base em objetivos estratégicos, usam seus exércitos em assuntos humanitários e reformulam seus interesses nacionais, graças ao amparo dos direitos humanos e à importância do ser humano em relação ao Estado. Para esse fim, os conceitos de segurança e poder da teoria realista são aplicados ao caso equatoriano, na experiência de missões de ajuda para reconstrução no Caribe: Marhec, Marcec e Mahrgec.
}

Palavras-chave: Estado; Forças Armadas; interesses; poder; segurança. 


\section{Introducción}

La existencia del Ejército -a partir de la teoría realista de relaciones internacionales - tiene como fundamento la necesidad de seguridad y poder; efectivamente, esta institución es la encargada de proteger la estabilidad y vigencia del Estado. Sin embargo, en el sistema internacional, así como existen Estados sin Ejército, hay algunos que destinan un presupuesto sumamente alto al mismo, tanto para defensa como para otras actividades vinculadas con el desarrollo. En este contexto, el presente artículo se circunscribe en el área de seguridad y, para contribuir a la explicación, se combina con el área de política exterior en lo relativo a Estados pequeños, desde una visión de realismo clásico.

En este artículo se busca responder a la pregunta: ¿̇uál es la vinculación de las Fuerzas Armadas con las situaciones de emergencia en desastres naturales?, específicamente, a la luz del caso ecuatoriano del envío de militares a tres misiones de ayuda para la reconstrucción ${ }^{1}$. Para ello, es preciso mencionar que existen dos posiciones: la primera acepta la presencia de los ejércitos en situaciones de emergencias en desastres naturales, principalmente, debido a su preparación y su capacidad para hacer frente a dichas situaciones; la segunda, cuestiona dicha presencia o vinculación, por considerarlos preparados para matar, lo que determina una preocupación por su contacto con la población civil. Adicionalmente, en términos prácticos, una visión más utilitaria podría señalar: si tenemos un ejército que puede ayudar, ipor qué no utilizarlo?; más aún, si no existe conflicto armado.

Respecto a la pregunta planteada, el argumento central de la visión realista es que los ejércitos no son una institución humanitaria, debido a que su composición, cadena de mando y competencias exclusivas los hacen necesarios para la defensa y seguridad del Estado. Sin embargo, según Morgenthau, puesto que el Estado se preocupa por los intereses y objetivos estratégicos basados en el posicionamiento y la reputación internacional, decide cooperar con otros Estados que atraviesan por una emergencia, y, para hacerlo, utiliza a sus ejércitos en asuntos humanitarios, a partir de la reformulación de intereses nacionales. A fin de justificar estas acciones racionales, el Estado aprovecha el valor internacional otorgado a los derechos humanos.

Para explicar dicha dinámica, se escogió el caso de Ecuador por las siguientes razones: a) la Constitución de la República

1 Ecuador, bajo la presidencia de Rafael Correa Delgado, envío contingente militar a Haití, Cuba y San Vicente y Granadinas, con la finalidad de contribuir a la emergencia que dichos Estados vivieron producto de los desastres naturales. El envío de militares a los Estados caribeños significó recursos para el Estado ecuatoriano, pues no únicamente se entregó material, sino que se hicieron obras y se contribuyó con el sector seguridad, especialmente en Haití. 
de Ecuador de 2008 incorporó la seguridad integral, que incluye la actuación estatal directa frente a los desastres naturales desde la gestión de riesgos; b) las Fuerzas Armadas ecuatorianas han participado en misiones de ayuda para la reconstrucción en desastres naturales, bajo un contexto de relación bilateral con los Estados beneficiarios; c) las Fuerzas Armadas, por disposición constitucional, son una institución garante de los derechos humanos.

A pesar de que Ecuador es considerado como un Estado pequeño en América Latina, desde sus inicios como república, e incluso desde antes de consolidarse como tal, ha tenido Ejército y permanentemente destina recursos al mantenimiento de sus Fuerzas Armadas. En un inicio, la principal preocupación de esta institución se centró en el conflicto territorial con sus vecinos, especialmente con Perú; no obstante, ha tenido además una fuerte vinculación con el desarrollo del país, especialmente en la época de las dictaduras militares y, después de la firma de la paz, en 1998, al vincularse con actividades relacionadas con la seguridad integral.

En virtud del argumento central del presente artículo, se escogió el realismo clásico de relaciones internacionales como una teoría que explica la relación de las Fuerzas Armadas con el Estado, los intereses y los objetivos estatales, así como la ayuda hacia otros Estados. Esta teoría permite comprender la participación de la institución militar, pues el nivel de análisis se centra en el Estado y atribuye la existencia de los ejércitos al "dilema de seguridad", según el cual la razón de ser de las Fuerzas Armadas es la presencia de enemigos estatales. Las preocupaciones centrales del realismo son la seguridad y el poder, en virtud las cuales los Estados racionales y unitarios protegen su soberanía y buscan maximizar sus intereses.

Si bien se considera que en las relaciones internacionales existen varias teorías, se ha descartado el liberalismo, pues deja de lado las capacidades militares y, en cambio, propone el desarme y el derecho como mecanismos para mantener la paz (David, 2008). Esta visión no contribuye a la explicación debido a que, aplicado al caso propuesto, Ecuador tiene Fuerzas Armadas dedicadas principalmente a la protección del territorio y de la soberanía.

Por otra parte, se descartan las "teorías críticas" pues, en su generalidad, sostienen que la seguridad es el producto de discursos dominantes que anticipan realidades; además, cuestionan la capacidad de los Estados para garantizar la seguridad a sus individuos y apuestan por la emancipación humana como el mecanismo para lograr una verdadera seguridad, en la medida en que consideran que el Estado es el que en realidad oprime y vulnera los derechos humanos (David, 2008).

Para el desarrollo del tema propuesto se empleó una "ontología objetivista", una "epistemología positivista" y una "metodología cualitativa" enfocadas en explicar la vinculación de los militares en asuntos humanitarios, tomando 
como caso de estudio a las Fuerzas Armadas del Ecuador. El método utilizado será, en esta medida, el estudio de caso. Respecto a la obtención de información para el desarrollo de la investigación, se utilizó el análisis documental, tanto de las constituciones como de las leyes específicas vinculadas con atribuciones y competencias de las Fuerzas Armadas ecuatorianas, documentos oficiales de la Función Ejecutiva ecuatoriana y notas de prensa oral y escrita. También, se recurrió a la investigación por internet y entrevistas semiestructuradas.

El desarrollo del presente artículo tiene tres momentos importantes: en un primer momento, se explica la existencia de las Fuerzas Armadas a la luz del realismo clásico en relaciones internacionales y su vinculación con la seguridad, bajo una perspectiva de Estados pequeños; en un segundo momento, se presenta una visión general del Ecuador como Estado pequeño y de las Fuerzas Armadas con sus misiones a través del tiempo; en un tercer momento, se abordan las misiones de ayuda para la reconstrucción en el Caribe (Marhec, Marcec y Mahrgec), así como los resultados obtenidos en ellas; finalmente, se presenta una conclusión obtenida de los resultados de la investigación aplicados al caso ecuatoriano.

\section{Las Fuerzas Armadas y la seguridad: visión desde el realismo clásico}

Hablar de Fuerzas Armadas necesariamente implica hablar de Estado. La definición de este último se ha deba- tido ampliamente, tanto en lo político como en lo jurídico; sin embargo, para efectos de este trabajo, se entenderá el Estado como una "comunidad de personas, asentada en un espacio físico claro y totalmente delimitado, intrínsecamente dotada de poder suficiente para organizarse y organizar la vida de todos sus elementos, proveerse de los medios necesarios para subsistir con independencia de todo poder extraño" (Trujillo Vásquez, 2006, p.53). Esta definición evidencia varios elementos necesarios para su existencia: territorio, población y autoridad, aunque la soberanía y el reconocimiento internacional son también elementos importantes.

Sobre la base de la definición de Estado, entendemos que el territorio y la soberanía son los fundamentos para la existencia de las fuerzas de seguridad: policía y militares, los cuales actúan en virtud de las decisiones de la autoridad del Estado, en defensa de este. La presencia de militares permite al Estado tener personas preparadas para la protección del territorio y de la soberanía dentro del sistema internacional anárquico. Dicho de otra forma, la protección del Estado, especialmente del territorio y de la soberanía, son las misiones específicas de las Fuerzas Armadas, puesto que, en el caso de que otro Estado se apodere de dichos elementos, se pone en peligro la existencia del Estado mismo.

¿Por qué cuando hablamos de Estado pensamos en seguridad? Esta pregunta se presenta cada vez que un Estado define sus competencias, establece 
qué entiende por seguridad y diseña sus políticas públicas. A partir de los postulados centrales del contrato social de Hobbes, se puede decir que: "la seguridad es el gran objetivo que empuja a los hombres a pactar para salir del estado de naturaleza y constituir la sociedad política y el Estado" (Rojas Aravena y Álvarez Marín, 2012, p.9). La seguridad es un bien preciado que debe ser encargado a un ente superior a la individualidad personal; de ahí que el Estado, al ostentar el uso legítimo de la fuerza, es el responsable de la seguridad de su población y de su defensa en cuanto actor en el sistema internacional.

Las teorías de relaciones internacionales buscan explicar cómo funciona el mundo, cuáles son los comportamientos de los Estados, qué los motiva a actuar de determinada forma y cómo explicamos el sistema internacional. En esta línea, la teoría realista considera al Estado como el principal actor del sistema, el cual tiene atributos que le permiten defenderse y existir en el sistema internacional.

El realismo clásico se basa en el pensamiento de Tucídides, Nicolás Maquiavelo, Thomas Hobbes, Edward Carr y Hans Morgenthau. Este enfoque dominó el pensamiento internacional después de la Segunda Guerra Mundial y centró su análisis en el poder en un sistema anárquico, no en el sentido de desorden, sino de ausencia de gobierno mundial. Para el realismo, los "Estados deben buscar maximizar el poder y proyectarlo en el ambiente externo para beneficio propio" (Velásquez Flores y González Cruz, 2014, p. 211), es decir, sus acciones se basan en las decisiones racionales y unitarias, razón por la cual "necesitan adquirir capacidades militares suficientes para inhibir cualquier ataque de un enemigo potencial" (p. 218).

Para el realismo existen temas de agenda más importantes que otros, por ello, diferencian entre high politics y low politics. En el primer grupo están los asuntos de seguridad y militares, lo que evidencia que los Estados no pueden entregar la tarea de seguridad a otros actores distintos; en el segundo grupo, aquellos temas de preocupación estatal que no tienen que ver con temas de seguridad, soberanía y asuntos militares. La seguridad, para el realismo, se enfoca en el Estado y en los intereses nacionales bajo el criterio de sobrevivencia y desconfianza. Por otra parte, considera al Estado como racional y unitario, es decir, que sus "acciones están basadas en los objetivos planteados, los intereses supremos de la nación y en los valores que busca proyectar hacia el exterior [...] [y] actúa como un solo ente en el escenario internacional" (Velásquez Flores y González Cruz, 2014, p.217).

El poder es otro concepto clave dentro del realismo, puesto que el Estado, en su racionalidad, "procura mantener poder, aumentar el poder o demostrar poder"(Morgenthau, 1986, p.63). Por ello, la solución para la anarquía del sistema es el equilibrio del poder. Los Estados aplican una "política exterior 
tendiente a conservar el poder y evitar el cambio de la distribución del poder en su favor"(Morgenthau, 1986, p.6364), así como una política de prestigio, en la medida de sus capacidades materiales, que les permita sobrevivir en el sistema. Esta política es utilizada de diversas maneras, por ejemplo, desde la existencia, preparación y mantenimiento de las fuerzas de seguridad y los aspectos centrales de la agenda de política exterior.

El estudio del equilibrio del poder evidencia la existencia de Estados desiguales; en ello radica la importancia de estudiar a los Estados pequeños, los cuales fueron relegados por varias décadas de los estudios internacionales, considerados como insignificantes. Sin embargo, como afirma Hey (2003), a partir de la Guerra Fría y del Golfo, el estudio de los Estados pequeños adquirió "mayor prestigio y visibilidad internacional que en cualquier otra época de la historia" (p.3).

Para definir a un small state es preciso considerar que las dos visiones centrales de pequeñez se centren en poder y capacidades materiales (Salgado, 2017, p.12), que pueden ser medidos en función de territorio, economía, aspectos políticos o militares, entre otros. De igual forma, la pequeñez de un Estado debe darse en relación con otro, pues en la individualidad no caben dichas determinaciones; por ello se indica que "la mayoría de los Estados pequeños se encuentran yuxtapuestos entre las grandes potencias o a la sombra de una gran potencia"
(Hey, 2003, p.186). Goetschel (1998) señala, respecto de la pequeñez de un Estado, que esta "depende de la noción de poder y de la naturaleza del sistema internacional" (p.14), pero no se aparta del todo de las capacidades materiales que condicionan las acciones y omisiones estatales.

Para el realismo clásico, un Estado pequeño es aquel que tiene capacidades materiales limitadas y que no amenaza la existencia y seguridad de sus vecinos. Con el fin de complementar el concepto de pequeñez, es importante considerar el criterio de percepción, tanto del Estado como de su población y de otros Estados (Hey, 2003, p.5). Este proceso de percepción implica que el Estado reconozca y asuma su déficit de autonomía, influencia y poder, con el fin de tomar decisiones que permitan disminuirlo o, al menos, mantenerlo para no amenazar su existencia (Goetschel, 1998, pp.13-14).

Los Estados, grandes y pequeños, se relacionan en el sistema internacional, por lo que es importante determinar la política exterior de los Estados pequeños. Con relación a la finalidad del presente trabajo, consideraremos que:

La política exterior es, a la vez, las tendencias generales de la conducta y las acciones particulares tomadas por un Estado u otro actor colectivo en su orientación hacia otros actores colectivos dentro del sistema internacional. Las acciones de política exterior pueden emprenderse utilizando una variedad 
de instrumentos diferentes, que van desde la adopción de declaraciones, la formulación de discursos, la negociación de tratados, la ayuda económica a otros estados, la participación en actividades diplomáticas como las cumbres y el uso de la fuerza militar (Beach, 2012, p.3).

Como se indicó, las decisiones, acciones y omisiones de los Estados forman parte de su política exterior, la cual se diseña sobre la base de criterios y directrices de Estado y/o gobierno; pero, en virtud de la racionalidad estatal, estas decisiones obedecen a intereses y objetivos estratégicos nacionales.

Para el realismo, la política exterior del Estado está centrada en la seguridad y el poder. Por ello, Wohlforth (2012) clasifica el comportamiento de los Estados en: grupismo, egoísmo y poder-centrismo (p.36). El primero, con miras a sobrevivir en el sistema internacional, hace que los Estados se cohesionen en función de sus intereses; el segundo sostiene que "el egoísmo tiende a superar al altruismo" (p.36); el tercero señala que la guía de toda decisión estatal siempre será el deseo de poder (p.36). No obstante, los Estados justifican sus decisiones en función de sus intereses nacionales, utilizando incluso temas de interés para la comunidad internacional, tales como: valores, soberanía, derechos humanos, independencia, entre otros.

Por otra parte, es necesario tener presente que en función de la racionalidad del Estado, para el realismo la cooperación no está del todo negada, puesto que la misma es viable siempre y cuando satisfaga los intereses estatales (Karns y Mingst, 2004, p.46). Así también, es preciso considerar que las decisiones de política exterior estatales son adoptadas bajo un esquema de sobrevivencia en el sistema, considerando las dificultades y condiciones del Estado. Por ello, como afirma Beach (2012), los Estados y por ende sus líderes adoptan "políticas que maximicen la capacidad del Estado para sobrevivir y preservar su autonomía independientemente de [su] ideología o personalidad" (p.18). En resumen, más allá de las justificaciones para las decisiones, acciones y omisiones del Estado, "el poder siempre será el objetivo inmediato" (Morgenthau, 1986, p.41).

Respecto al caso de los Estados pequeños, cabe la apreciación de que el conocimiento interno de la pequeñez y las circunstancias internacionales influyen en la política exterior. En este sentido:

El entorno internacional ofrece a los pequeños Estados nuevas oportunidades para defender [sus] intereses [...], por lo que su tamaño no tiene un impacto negativo a priori. [Pero] Los cambios en este entorno, así como la modificación de las políticas aplicadas por los pequeños Estados, pueden modificar el déficit de poder relativo de estos estados, haciéndolos menos pequeños desde el punto de vista tradicional. (Goetschel, 1998, p.30)

Si bien las condiciones materiales influyen en el concepto de pequeñez, el 
diseño y ejecución de una política exterior coherente con la realidad y con los objetivos estratégicos es importante para incrementar o, al menos, mantener el poder del Estado y sobrevivir en el sistema internacional.

El aprovechamiento del entorno y el conocimiento interno de las condiciones del Estado llevan a que la política exterior de un Small State sea bastante diversa. Por ello, los Estados pequeños pueden conservar la neutralidad o adoptar acciones y decisiones positivas o negativas con mayor riesgo, así como justificarlas en virtud de su condición y necesidad de prestigio a la luz del sistema (Hey, 2003, pp.10-11). Por otro lado, es preciso considerar que el nivel de análisis permite realizar la política exterior estatal, puesto que el Estado está limitado por "factores sistémicos (...) el nivel de desarrollo influye en el impacto de ciertos factores nacionales e internacionales (...) [y] el nivel de desarrollo influye en el papel del líder"(Hey, 2003, p.192), que decide en virtud de la racionalidad del Estado.

Por otro lado, respecto a la vinculación entre el concepto de prestigio y el de poder, el realismo clásico propone que la "aspiración al reconocimiento social es una poderosa fuerza dinámica que determina las relaciones sociales" (Morgenthau, 1986, p.100). Esto lleva a considerar que el Estado pequeño, en su afán de poder, utiliza el prestigio como estrategia para ser más visible e importante en el sistema, dada su condición de pequeñez. Por ello, el reconocimiento y el posicionamiento internacionales refuerzan la política de prestigio estatal, ya que "a través de su reputación de excelencia puede lograr la medida de seguridad, prosperidad y poder que estima le es debida" (Morgenthau, 1986, p.100). Para ejecutar esta política, los Estados se sirven de sus capacidades materiales, diplomacia y capacidad militar.

La seguridad, como se ha dicho, es uno de los grandes temas de interés para el Estado desde la visión realista; de hecho, la seguridad estatal es el fundamento de la existencia y el mantenimiento de las fuerzas de seguridad. Sin embargo, alejándonos un poco del concepto tradicional de guerra, las nuevas dinámicas mundiales han llevado al cuestionamiento de la necesidad de las Fuerzas Amadas en los Estados, principalmente por la ausencia de guerras interestatales. Esta visión ha llevado a que muchos Estados utilicen al personal militar en aspectos no tradicionales de la misión militar, por ejemplo, en la lucha contra el narcotráfico, la militarización de la seguridad pública (Grosso, 2012, p.98) y la intervención en desastres naturales. Estos usos, ajenos a la misión tradicional de defensa territorial, cuestionan "si es correcto afirmar que lo que está en juego en el ámbito militar es menos importante que antes y que la seguridad del Estado está menos determinada por los problemas militares en otras actividades" (David, 2008, p.93).

El concepto de seguridad se ha ido redefiniendo a través del tiempo, considerando las circunstancias mundiales, 
regionales y nacionales. Sin embargo, para efectos del presente trabajo, la seguridad es considerada como "la ausencia de amenazas militares y no militares que pueden cuestionar los valores centrales que quiere promover o preservar una persona o una comunidad y que conllevan un riesgo de utilización de la fuerza" (David, 2008, p.65). La seguridad relacionada con lo humanitario debe ser entendida de acuerdo con tres conceptos: internacional, nacional y humana. Esta última es considerada como el fundamento de la vinculación de las fuerzas de seguridad estatales con las acciones relacionadas con desastres naturales. Como afirma Fuentes (2012), la seguridad humana:

[...] enfatiza en la complejidad y la interrelación de las amenazas para la seguridad, tanto conocidas como nuevas, que van desde la pobreza crónica y persistente hasta la violencia étnica, pasando por el tráfico de personas, el cambio climático, las pandemias, el terrorismo internacional y el empeoramiento repentino de la situación económica y financiera (p. 33).

La seguridad humana amplía la visión de seguridad centrada en el Estado, la cual es beneficiosa porque permite abordar áreas no tradicionales de dicha temática. Sin embargo, la amplitud del concepto representa inconvenientes en tanto "coloca al Estado ante el escrutinio crítico, que permite juzgar sus políticas, sus capacidades, sus relaciones con los ciudadanos e incluso su legitimidad" (Pérez de Armiño, 2006, p.68). Esta situación, especialmente respecto al tema de desastres naturales, coloca al Estado como principal responsable de atender las emergencias generadas por efectos de la naturaleza, por lo que la comunidad internacional es responsable únicamente cuando el Estado no se encuentre en condición de responder adecuadamente.

La seguridad humana se presenta como justificación a las acciones humanitarias de las fuerzas de seguridad estatales, que en dicha medida pueden tener presencia tanto a nivel nacional como internacional, y no ser entendidas como intervención, dada la naturaleza de protección estatal y la misión de la institución armada. La justificación estatal para el empleo de fuerzas de seguridad desde esta visión tiene un triple objetivo: a) justificar el gasto estatal para el mantenimiento de ejércitos e instituciones de seguridad; b) aplicar políticas específicas encaminadas a alcanzar objetivos estratégicos; c) aplicar una política de prestigio para el Estado. Estos objetivos, desde la óptica del Estado pequeño realista, implican la utilización de militares a través de una justificación que esté ligada a los derechos humanos, la dignidad humana y la seguridad como deber estatal.

El empleo de militares en situaciones de emergencia tiene además una aplicación práctica, ya que "las FF.AA. disponen de capacidades, derivadas de su misión, que no están siendo utilizadas en operaciones reales" (Vivas, 2015, p.376), pero que pueden ser úti- 
les en situaciones adversas. Los buenos o malos resultados de esta aplicación militar tienen relación directa con el mandato, cumplimiento e interacción de los militares con los civiles, que implican necesariamente el respeto a los derechos humanos. En resumen, "la función humanitaria de los militares se ha visto además como una forma de potenciar la reputación y los recursos de unas fuerzas armadas sometidas a presiones fiscales internas y un apoyo público" (Oxfam, 2011, p.27).

\section{Ecuador: un Estado pequeño con fuerzas armadas permanentes}

A fin de determinar y comprender las misiones de las Fuerzas Armadas ecuatorianas, es preciso ahondar en la condición del Ecuador de ser un Estado pequeño. El planteamiento teórico del presente artículo incluyó el concepto de Estado pequeño desde la visión realista, ligada a las capacidades materiales y al poder. En este sentido, Ecuador es catalogado como tal por razones históricas vinculadas con "su ubicación en la conflictiva región andina (...) por la influencia estructural de los Estados Unidos y las interacciones con los países vecinos" (Bonilla, 2006, p.166), además de su territorio y aspectos económicos.

El territorio, como uno de los elementos del Estado, juega un rol importante en la determinación de la política exterior, puesto que permite conocer las fortalezas y debilidades para encaminar las decisiones que impliquen sobrevivir en el sistema internacional. La delimitación y protección del territorio le permite al Estado tener un "espacio del universo que es suyo, ya que dentro de este espacio es donde debe cumplir sus funciones y crear las condiciones para que sus ciudadanos gocen de los derechos que les son reconocidos" (Trujillo, 2006, p.61).

La situación territorial ecuatoriana, desde su creación como Estado, ha mantenido una tónica de conflicto con los Estados vecinos, Colombia y Perú, especialmente con esté último. El conflicto territorial inició por dos razones fundamentales: a) fronteras indeterminadas, y b) dudas respecto de la aplicación de los instrumentos jurídicos determinantes de la frontera estatal (Trujillo, 2006, p.63). Sin embargo, es preciso señalar que Ecuador heredó conflictos territoriales desde la Gran Colombia.

El mayor conflicto debido al territorio se debió a la intención ecuatoriana de tener acceso al Río Amazonas, no solo por un tema de extensión territorial en la zona oriental, sino principalmente por aspectos económicos. Para solucionar dichos inconvenientes, se suscribieron varios convenios internacionales a lo largo de los años. Esta preocupación del Estado llevó a que la política exterior se centre en el aspecto territorial. Uno de los instrumentos jurídicos más debatidos y que implicó grandes esfuerzos estatales es el Protocolo de Río de Janeiro (Bonilla, 1997, p.69). 
El conflicto territorial entre Ecuador y Perú, a lo largo de los años, tuvo reveces y adelantos diplomáticos, pero principalmente intervención militar en la zona de frontera (Ministerio de Relaciones Exteriores, 1995). Esta situación llevó a que los esfuerzos estatales de política exterior se encaminaran al mantenimiento del Ejército y a la solución del conflicto territorial. Incluso el conflicto territorial tuvo un impacto en el sentir de la población ecuatoriana, debido a que reforzó el sentimiento de identidad y unidad nacional, así como la aceptación de la labor militar. Al respecto, Carranza (2002) señala que:

La imagen del Ecuador o del Perú se crea cuando se hace referencia a los signos nacionales: la frontera, la historia o el objetivo nacional de reivindicación de un acceso al Amazonas1995 (p.142).

Después de la firma de la paz, en 1998, se puso fin al conflicto territorial entre Ecuador y Perú. En la actualidad, Ecuador es un Estado con una extensión ${ }^{2}$ de $256.370 \mathrm{~km}^{2}$, con una población de 17.120.729 ecuatorianos ${ }^{3}$; cifra que, frente a sus vecinos Colombia y Perú, lo hacen un Estado pequeño.

Los aspectos militares ecuatorianos tienen relación directa con la defensa del territorio, lo cual explica la atención del Estado y consecuentemente de los gobiernos en el mantenimiento del Ejército. La otra determinación que hace del Ecuador un Estado pequeño es la capacidad militar. Las Fuerzas Armadas ecuatorianas están compuestas por tres fuerzas: terrestre, aérea y naval, las cuales están actualmente bajo la dirección del Ministerio de Defensa en el aspecto político, y del Comando Conjunto de las FF.AA. en el aspecto técnico.

De conformidad con el Plan Nacional de Seguridad Integral, las Fuerzas Armadas están preparadas para defender al Estado de agresiones externas y proteger la soberanía e integridad territorial, $y$, en tiempos de paz, son responsables de "prevenir conflictos armados y proteger derechos, libertades y garantías de las y los ecuatorianos"(Ministerio Coordinador de Seguridad, 2014, p.33). Es preciso mencionar que la defensa nacional está encargada de:

[...] el fortalecimiento de las capacidades militares, manteniendo la subordinación a la autoridad política legalmente constituida, e interactúa en coordinación con la política exterior del Estado, colaborando en el mantenimiento de la paz y la seguridad internacionales y en el apoyo a las acciones del Estado en el ámbito interno. La actitud estratégica del Ecuador es defensiva y su orientación es proactiva. Se fundamenta en la prevención y alerta temprana y contempla como recurso de última instancia el empleo de

2 Recuperado de: http://www.cancilleria.gob.ec/bienvenidos-geografia-del-ecuador/

3 Recuperado de: http://www.ecuadorencifras.gob.ec/estadisticas/ (Corte al 22 de noviembre de 2018) 
la fuerza militar (Ministerio Coordinador de Seguridad, 2014, p.34).

En los últimos años, Ecuador emprendió reformas al sector seguridad, especialmente en el manejo del presupuesto destinado a salarios de militares y policías, bajo una política de mejora- miento de las condiciones de vida de estos sectores. Esto se evidenció en el incremento progresivo de las remuneraciones, tanto de la tropa como de los oficiales, y en las misiones realizadas en el marco de la seguridad integral, por ejemplo, el combate al narcotráfi$\mathrm{CO}$, conforme se muestra en la tabla 1 :

Tabla 1. Presupuesto de Ecuador al Sector Defensa, 2006-2016

\begin{tabular}{|l|l|l|l|l|}
\hline & \multicolumn{1}{|c|}{$\mathbf{2 0 0 6}$} & \multicolumn{1}{c|}{$\mathbf{2 0 1 0}$} & \multicolumn{1}{c|}{$\mathbf{2 0 1 4}$} & \multicolumn{1}{c|}{$\mathbf{2 0 1 6}$} \\
\hline Ecuador & 952.621 .138 & 2.156 .832 .116 & 2.773 .004 .221 & 2.510 .507 .785 \\
\hline Colombia & 2.872 .392 .573 & 6.178 .26 .917 & 8.416 .388 .574 & 4.916 .946 .842 \\
\hline Perú & 1.086 .270 .304 & 2.061 .617 .832 & 2.819 .591 .821 & 2.237 .685 .498 \\
\hline Uruguay & 215.709 .213 & 622.039 .810 & 650.718 .647 & 770.840 .944 \\
\hline
\end{tabular}

Fuente: elaboración propia a partir de los datos contenidos en el Atlas Comparativo de la Defensa en América Latina y Caribe, edición 2016.

Tabla 2. Cuadro comparativo de remuneraciones militares periodo 2006-2018

\begin{tabular}{|l|c|c|c|}
\hline \multicolumn{4}{|c|}{ Gemuneraciones de servidores militares (comparativo) } \\
\hline General de división & $\begin{array}{c}\text { 2006 Remuneración no } \\
\text { homologada }\end{array}$ & $\begin{array}{c}\text { Remuneración } \\
\text { homologada }^{\mathbf{4}}\end{array}$ & Remuneración 2018 \\
\hline General de brigada & $\$ 2.149$ & $\$ 3.885$ & $\$ 5.358$ \\
\hline Teniente coronel & $\$ 1.856$ & $\$ 3.220$ & $\$ 5.111$ \\
\hline Mayor & $\$ 1.139$ & $\$ 2.250$ & $\$ 3.541$ \\
\hline Suboficial & $\$ 929$ & $\$ 1.230$ & $\$ 2.944$ \\
\hline Sargento primero & $\$ 819$ & $\$ 1.092$ & $\$ 2.669$ \\
\hline Soldado & $\$ 278$ & $\$ 734$ & $\$ 1.643$ \\
\hline
\end{tabular}

Fuente: elaboración propia a partir de información del diario El Universo (02 de junio de 2006$)^{5}$ y de escala remunerativa de militares (fuerza terrestre), vigente a 2018.

Los cuadros presentados reflejan que Ecuador, pese a su población, destina presupuesto al sector defensa, el cual se ha incrementado a lo largo de los años. Esto llama la atención porque, si bien el Estado ya no tiene un conflicto

4 Cabe indicar que las remuneraciones de los militares se fueron ajustando en cuatro ejercicios fiscales (2007-2010).

5 "Las FF.AA. con alzas de hasta 80\% en sueldos" (02 de junio de 2006). Diario El Universo. https://www. eluniverso.com/2006/06/02/0001/9/E3800CAC13954B10B5C73D8F28C8881D.htmINO ESTÁ EN LAS REFERENCIAS BIBLIOGRÁFICAS. 
directo como en décadas pasadas, la defensa de la soberanía no se ha dejado de lado. Así mismo, cabe señalar que, conforme a los datos del Comparativo de la Defensa en América Latina y el Caribe, Ecuador tiene 25 militares por cada 10.000 habitantes, y que esta carrera militar, al igual que las relacionadas con la seguridad ciudadana, son atractivas para la población, especialmente por la estabilidad laboral.

El último aspecto de las condiciones materiales que catalogan a Ecuador como un Estado pequeño es el factor económico. Al respecto, la economía ecuatoriana ha tenido etapas de bonanza y de crisis, ejemplos de ello son: el boom cacaotero, el boom petrolero y la crisis del dólar. Así mismo, el factor económico es decisivo entre la sierra y la costa debido al regionalismo. De hecho, en las primeras décadas de la república, estas pugnas de poder entre el sector agrícola de la sierra y la costa, y la banca llevaron al Estado a perder el control de la política monetaria (De la Torre Arauz, 2013). Después de la caída del precio del cacao, el Estado dependió "del sector agroexportadorbancario para cubrir gastos administrativos, como el pago a la burocracia civil y militar" (2013, p.108), hasta la época en que se realizaron reformas en política monetaria, con la creación del Banco Central, durante la década de los 20 del siglo pasado.
Por otra parte, en temas económicos Ecuador ha sido tradicionalmente un Estado dependiente de Estados Unidos, el cual ha orientado "medidas políticas económicas de ajuste estructural orientadas a modernizar [las] feudales formas de producción" (De la Torre Arauz, 2013, p.123). La aplicación de recetas internacionales y la ausencia del Estado en varios temas de interés nacional como los sectores estratégicos, determinaron que su desarrollo económico esté "sustentado en exportaciones primarias (primero agrícolas y luego minerales)"(Senplades, 2009, p.31).

En este contexto, las Fuerzas Armadas han estado presentes en todas las épocas del país y formado parte trascendental de la evolución estatal, pues su presencia no solo ha sido en el sector defensa, sino también en los sectores político y económico. Esta vinculación estrecha de los militares con la ciudadanía se produjo tanto por el conflicto territorial con Perú como por la participación en situaciones políticamente adversas, pues mientras "otras instituciones del Estado desatendían necesidades de la sociedad, los militares eran llamados a solucionar dichas exigencias" ${ }^{\prime 6}$

Respecto a las misiones de las Fuerzas Armadas, es preciso mencionar que estas se han establecido en todas las constituciones ecuatorianas, desde la

6 "Las Fuerzas Armadas ecuatorianas son un reflejo de la sociedad" (12 de marzo de 2016). El Telégrafo. Recuperado de: https://www.eltelegrafo.com.ec/noticias/politica/3/las-fuerzas-armadas-ecuatorianas-sonun-reflejo-de-la-sociedad No está en el listado final de referencias bibliográficas. 
formación del Estado ecuatoriano. La primera Constitución (Congreso Constituyente, 1830) creó al Ejército con la misión de "defensa de la independencia de la patria, de sus leyes y el mantenimiento del orden público" (Macías, 2010, p.252). Sobre esta creación institucional se hicieron reformas estructurales, tanto en el sistema de formación como de ingreso al Ejército, principalmente en los gobiernos de $\mathrm{Vi}$ cente Rocafuerte, Gabriel García Moreno y Eloy Alfaro; en la época de la Revolución Juliana; de Isidro Ayora y Velasco Ibarra, así como en las dictaduras militares de los años 70. En este sentido, las Fuerzas Armadas, a través de las tres fuerzas (terrestre, naval y aérea), se han centrado en "proteger a la nación, rechazando toda acción de fuerza en contra de los derechos individuales y colectivos del pueblo, apoyar a la política nacional en el logro de sus objetivos y contribuir así a la seguridad del Estado" (Moncayo, 1994, p.91).

A lo largo de la época republicana, Ecuador ha tenido veinte constituciones, las cuales han establecido misiones específicas para las Fuerzas Armadas; sin embargo, se han mantenido siempre la protección del territorio, la soberanía y la defensa nacional, conforme consta en la tabla 3:

Tabla 3. Misiones de las FF.AA, 1830-2008

\begin{tabular}{|l|l|}
\hline \multicolumn{1}{|c|}{ Constitución } & \multicolumn{1}{|c|}{ Misiones, atribuciones y competencias } \\
\hline Constitución de $1830^{7}$ & $\begin{array}{l}\text { Defender la independencia de la patria. } \\
\text { Sostener las leyes. } \\
\text { Mantener el orden público. }\end{array}$ \\
\hline Constitución Política de $1835^{8}$ & $\begin{array}{l}\text { Defensa exterior del Estado. } \\
\text { Conservar del orden interior. }\end{array}$ \\
\hline Constitución Política de $1843^{9}$ & Defensa y seguridad del Estado. \\
\hline Constitución Política de $1845^{10}$ & $\begin{array}{l}\text { Defensa y seguridad de la República. } \\
\text { Mantener o restablecer el orden y tranquilidad } \\
\text { de la República. } \\
\text { Defensa exterior. } \\
\text { Conservación del orden interior. }\end{array}$ \\
\hline Constitución Política de $1851^{11}$ & $\begin{array}{l}\text { Defender la independencia y dignidad de la } \\
\text { República contra ofensas y agresiones exteriores. } \\
\text { Mantener el orden interno. } \\
\text { Asegurar la ejecución de leyes. }\end{array}$ \\
\hline Constitución de la República del Ecuador de $1852^{12}$ & $\begin{array}{l}\text { Defensa exterior del Estado enfocada en la } \\
\text { independencia y libertad de la República. }\end{array}$ \\
\hline Constitución de la República del Ecuador de $1861^{13}$ & Defender la República. \\
\hline
\end{tabular}

7 Artículo 51 (Congreso Constituyente, 1830).

8 Artículo 85 (Congreso Constitucional, 1835).

9 Artículo 60 numeral 4 (Convención Nacional, 1843).

10 Artículo 70 numeral 4 y artículo 102 (Convención Nacional, 1845) No se publicó en el RO.

11 (Convención Nacional) No se publicó en el RO.

12 Artículos 101 y 104 (Asamblea Nacional, 1852).

13 Artículo 99 (Convención Nacional, 1861). 


\begin{tabular}{|c|c|}
\hline Constitución del Ecuador de $1869^{14}$ & Defender la República. \\
\hline $\begin{array}{l}\text { Constitución Política de la República del Ecuador de } \\
1878^{15}\end{array}$ & $\begin{array}{l}\text { Defender la soberanía e independencia de la } \\
\text { República. }\end{array}$ \\
\hline Constitución Política del Ecuador de $1884^{16}$ & Defender la República. \\
\hline $\begin{array}{l}\text { Constitución Política de la República del Ecuador de } \\
1897^{17}\end{array}$ & Defender la República. \\
\hline $\begin{array}{l}\text { Constitución Política de la República del Ecuador de } \\
1906^{18}\end{array}$ & Asegurar los derechos de la nación en el exterior. \\
\hline $\begin{array}{l}\text { Constitución Política de la República del Ecuador de } \\
1929^{19}\end{array}$ & $\begin{array}{l}\text { Defender la nación. } \\
\text { Garantizar la ejecución de las leyes. }\end{array}$ \\
\hline Constitución Política de $1945^{20}$ & $\begin{array}{l}\text { Conservar la soberanía nacional. } \\
\text { Defensa de la integridad e independencia de la } \\
\text { República. } \\
\text { Garantizar el cumplimiento de la Constitución y } \\
\text { de las leyes. }\end{array}$ \\
\hline $\begin{array}{l}\text { Constitución Política de la República del Ecuador de } \\
1946^{21}\end{array}$ & $\begin{array}{l}\text { Defender la República. } \\
\text { Mantener el orden constitucional. }\end{array}$ \\
\hline Constitución de la República del Ecuador de $1967^{22}$ & $\begin{array}{l}\text { Asegurar la soberanía del Estado. } \\
\text { Garantizar el orden constitucional. } \\
\text { Defender de ataques externos a la Nación. }\end{array}$ \\
\hline Constitución Política del Ecuador de $1979^{23}$ & $\begin{array}{l}\text { Conservar la soberanía nacional. } \\
\text { Defender la integridad e independencia del } \\
\text { Estado. } \\
\text { Garantizar el ordenamiento jurídico. }\end{array}$ \\
\hline $\begin{array}{l}\text { Constitución Política de la República del Ecuador de } \\
1998^{24}\end{array}$ & $\begin{array}{l}\text { Conservar la soberanía nacional. } \\
\text { Defender la integridad e independencia del } \\
\text { Estado. } \\
\text { Garantizar el ordenamiento jurídico. }\end{array}$ \\
\hline Constitución de la República del Ecuador $2008^{25}$ & $\begin{array}{l}\text { Proteger derechos, libertades y garantías de los } \\
\text { ciudadanos. } \\
\text { Defender la soberanía e integridad territorial. } \\
\text { Apoyar en la seguridad integral del Estado }{ }^{26} \text {. }\end{array}$ \\
\hline
\end{tabular}

Fuente: elaboración propia a partir de la revisión de los textos constitucionales, desde la Constitución de 1830 hasta la de 2008 y sus reformas posteriores.

14 Artículo 84 (Convención Nacional, 1869).

15 Artículo 107 (Asamblea Nacional, 1878).

16 Artículo 121 (Asamblea Nacional del Ecuador, 1884).

17 Artículo 125 (Asamblea Nacional, 1897).

18 Artículo 117 (Asamblea Nacional, 1906).

19 Artículo 145 (Asamblea Nacional, 1929).

20 Artículo 113 (Asamblea Nacional Constituyente, 1945).

21 Artículo 153 (Asamblea Constituyente, 1946).

22 Artículo 248 (Asamblea Nacional Constituyente, 1967).

23 Artículo 128 (Consejo Supremo de Gobierno, 1979).

24 Artículo 183 (Asamblea Nacional Constituyente, 1998).

25 Artículos 158, 162 (Asamblea Nacional Constituyente, 2008)

26 En agosto de 2018, la Corte Constitucional del Ecuador declaró la inconstitucionalidad por la forma de la votación de las enmiendas constitucionales de 2015. Esta determinación significó que el apoyo de las Fuerzas Armadas en la seguridad integral no se encuentra vigente. No obstante, la Ley de Seguridad Pública y del Estado, reformada en el 2014, prevé dicha competencia. 
Conforme consta en la Constitución ecuatoriana, la Policía Nacional y las Fuerzas Armadas, más allá de sus misiones específicas, son instituciones protectoras de derechos, libertades y garantías de los ciudadanos. La relación de estas instituciones armadas, en concordancia con los conceptos de seguridad humana y seguridad integral, y bajo las directrices de política exterior previstas en la Constitución de la República, fueron el antecedente para el envío de misiones de ayuda para la reconstrucción ecuatoriana en el Caribe.

\section{Las misiones ecuatorianas de ayuda para la reconstrucción en el Caribe}

David (2008) destaca la importancia del rol de las Fuerzas Armadas, que tradicionalmente se dedicaron a la seguridad externa, e incluso fueron también garantes de la democracia en Ecuador, lo cual les permitió actuar en la política del país. Esta vinculación y aceptación política permitió que en el gobierno del expresidente Rafael Correa Delgado, en el marco de la cooperación bilateral, se enviaran militares y destinaran recursos para la reconstrucción de las zonas afectadas por los desastres naturales en el Caribe.

Esta decisión política se enmarcó en el principio de legalidad que rige a la función pública, en los principios de relaciones internacionales y en los planes nacionales de desarrollo y de seguridad integral que rigen la política pública y el presupuesto nacional (Asamblea Nacional Constituyente, 2008). El Plan Nacional de Seguridad
Integral de la época en que se enviaron las misiones de ayuda para la reconstrucción define a la seguridad como la “condición en que no existe peligro de ataque militar, presión política, coerción económica o ninguna imposición externa en la toma de decisiones concernientes a los asuntos del Estado, de manera que pueda seguir libremente su propio desarrollo y progreso" (Ministerio Coordinador de Seguridad, 2014, p.33).

La definición de seguridad aportó la justificación técnica para el envío de militares en un contexto de cooperación bilateral que no implicara presión política en los Estados beneficiados, sumado a lo establecido en la Constitución ecuatoriana vigente respecto de la política exterior, en la que se señala que uno de sus objetivos es "garantizar la soberanía nacional, promover la integración latinoamericana e impulsar una inserción estratégica en el contexto internacional, que contribuya a la paz y a un sistema democrático y equitativo mundial" (Asamblea Nacional Constituyente, 2008). Cabe indicar que las decisiones de política exterior, seguridad y defensa son competencias exclusivas del Estado Central, y las entidades de seguridad, Fuerzas Armadas y Policía Nacional dependen de la Función Ejecutiva ecuatoriana, las cuales, por su naturaleza, son obedientes a las decisiones legales y legítimas de sus autoridades.

Las misiones abordadas en el presente artículo tuvieron lugar en Haití, Cuba y 
San Vicente y Granadinas. Estas misiones se caracterizaron por:

a. Tener prioridad de inversión de la Secretaría Nacional de Planificación y Desarrollo (Senplades), ya que implicaron recursos públicos.

b. Haber sido ejecutadas al amparo de la Constitución de la República de 2008.

c. Ser ejecutadas por las Fuerzas Armadas, específicamente por el Cuerpo de Ingenieros del Ejército, bajo la dirección de la Fuerza Terrestre, el Comando Conjunto de las Fuerzas Armadas, el Ministerio de Defensa y el Ministerio de Desarrollo Urbano y Vivienda (Miduvi).

d. Tener como antecedente un desastre natural.

e. Ser negociadas de manera bilateral y directa entre el Estado ecuatoriano y los tres Estados beneficiados.
Las misiones de Apoyo a la Reconstrucción de Cuba - Ecuador (Marcec), de Ayuda Humanitaria para la Reconstrucción de las Granadinas- Ecuador (Mahrgec), y, de Apoyo a la Reconstrucción de Haití - Ecuador (Marhec), tuvieron participación de personal militar directivo y técnico. Este personal fue escogido y preparado de manera específica debido a que las misiones eran extranjeras, el personal iba a estar lejos de su familia y el trabajo implicaba contacto directo con personal civil en situación de vulnerabilidad (Peña Cobeña, 2018). El personal enviado cumplió con las directrices y normas de los soldados de paz que operan bajo directrices de la Organización de Naciones Unidas, especialmente por el componente de derechos humanos (Navarrete, 2018).

A fin de resumir las misiones propuestas, se muestra el siguiente cuadro:

Tabla 4. Misiones de reconstrucción en el Caribe

\begin{tabular}{|l|l|l|l|}
\hline & \multicolumn{1}{|c|}{ Marcec } & \multicolumn{1}{c|}{ Mahrgec } & \multicolumn{1}{c|}{ Marhec } \\
\hline Antecedente & \multicolumn{1}{|c|}{ Huracán Sandy, 2012 } & Tormenta Bertha, 2013 & \multicolumn{1}{c|}{ Terremoto, 2010 } \\
\hline \multirow{5}{*}{ Documento } & $\begin{array}{l}\text { Acuerdo de Cooperación } \\
\text { Técnica, Económica y } \\
\text { Científica entre Ecuador y } \\
\text { Cuba, suscrito en 2001 y } \\
\text { ratificado en 2013 (Miduvi, } \\
\text { 2013). }\end{array}$ & $\begin{array}{l}\text { Visita del Primer Ministro de } \\
\text { San Vicente y Granadinas, } \\
\text { Ralph Gonsalves por temas } \\
\text { políticos y de cooperación. }\end{array}$ & \\
\hline & $\begin{array}{l}\text { Miduvi, Ministerio de } \\
\text { Finanzas, Ministerio de } \\
\text { Defensa y Comando } \\
\text { Conjunto de las Fuerzas } \\
\text { Armadas. }\end{array}$ & $\begin{array}{l}\text { Ministerio de Defensa y } \\
\text { Comando Conjunto de las } \\
\text { Fuerzas Armadas }\end{array}$ & $\begin{array}{l}\text { Ministerio de Defensa y } \\
\text { Comando Conjunto de las } \\
\text { Fuerzas Armadas }\end{array}$ \\
\hline \multirow{2}{*}{ Ejecución } & $\begin{array}{l}\text { Cuerpo de Ingenieros del } \\
\text { Ejército }\end{array}$ & $\begin{array}{l}\text { Cuerpo de Ingenieros del } \\
\text { Ejército }\end{array}$ & $\begin{array}{l}\text { Cuerpo de Ingenieros del } \\
\text { Ejército }\end{array}$ \\
\hline
\end{tabular}




\begin{tabular}{|l|l|l|l|}
\hline & & & $\begin{array}{l}\text { Desarrollo en 4 compañías: } \\
\text { Marhec I, II, III y IV. Las } \\
\text { compañías incluyeron: } \\
\text { construcción de puentes } \\
\text { y vivienda, ampliación } \\
\text { de vías, capacitación } \\
\text { en el sector seguridad, } \\
\text { limpieza de canales } \\
\text { de riego y drenaje, así } \\
\text { como construcción y } \\
\text { rehabilitación de unidades } \\
\text { educativas y de salud. }\end{array}$ \\
\hline $\begin{array}{l}\text { Médicas de la Universidad } \\
\text { de Santiago de Cuba, y } \\
\text { construir 560 unidades } \\
\text { habitacionales en el distrito } \\
\text { de Abel Santamaría. }\end{array}$ & $\begin{array}{l}\text { Construcción de cuatro } \\
\text { puentes (Hope, Top } \\
\text { Sharpes, Chateabelair y } \\
\text { Kakarta). }\end{array}$ & años \\
\hline Duración & 3 años & 8 meses & \$30`249.823,45 USD \\
\hline Costo & $\$ 19.034 .058,51$ USD & $3.861 .680,23$ USD & \\
\hline
\end{tabular}

Fuente: elaboración propia a partir de la revisión de los documentos oficiales de priorización de inversión, contratos y proyectos presentados, así como de instrumentos de cooperación internacional.

\section{Respecto de los logros y beneficios, así frentó el Estado ecuatoriano al enviar como de los desafíos que obtuvo y en- tropas, se indica:}

Tabla 5. Logros y desafíos para Ecuador en las misiones Marcec, Mahrgec y Marhec

\begin{tabular}{|c|c|c|c|c|}
\hline Misión & \multicolumn{2}{|r|}{ Logros } & \multicolumn{2}{|r|}{ Desafíos } \\
\hline \multirow{9}{*}{$\begin{array}{l}\text { Marcec } \\
\text { Mahrgec } \\
\text { Marhec }\end{array}$} & \multirow{6}{*}{ Políticos } & $\begin{array}{l}\text { Cumplimiento del objeto de las } \\
\text { misiones. }\end{array}$ & \multirow{6}{*}{ Políticos } & $\begin{array}{l}\text { Negociación en la } \\
\text { adquisición de bienes y } \\
\text { servicios locales. }\end{array}$ \\
\hline & & $\begin{array}{l}\text { Reconocimiento de los Estados } \\
\text { beneficiados por el apoyo } \\
\text { brindado. }\end{array}$ & & $\begin{array}{l}\text { Negociación en aspectos } \\
\text { aduaneros. }\end{array}$ \\
\hline & & Generación de empleo local. & & \multirow{2}{*}{$\begin{array}{l}\text { Mantener prioridad de los } \\
\text { proyectos de inversión para } \\
\text { reconstrucción }\end{array}$} \\
\hline & & $\begin{array}{l}\text { Apoyo al desarrollo social, } \\
\text { productivo y de planificación. }\end{array}$ & & \\
\hline & & $\begin{array}{l}\text { Incremento de credibilidad en el } \\
\text { accionar de las Fuerzas Armadas. }\end{array}$ & & \multirow{2}{*}{$\begin{array}{l}\text { Negociación respecto de } \\
\text { manejo de personal de las } \\
\text { misiones y beneficios en } \\
\text { Estados beneficiados. }\end{array}$} \\
\hline & & $\begin{array}{l}\text { Visión de Ecuador como Estado } \\
\text { solidario. }\end{array}$ & & \\
\hline & \multirow{3}{*}{ Operativos } & $\begin{array}{l}\text { Rapidez en la priorización de } \\
\text { inversión y ejecución debido } \\
\text { a la naturaleza bilateral de la } \\
\text { cooperación }\end{array}$ & \multirow{3}{*}{ Operativos } & $\begin{array}{l}\text { Demora en transferencias } \\
\text { y pagos al personal que } \\
\text { participó en las misiones. }\end{array}$ \\
\hline & & $\begin{array}{l}\text { Transferencia de conocimientos } \\
\text { al personal militar. }\end{array}$ & & \multirow{2}{*}{$\begin{array}{l}\text { Selección de personal } \\
\text { idóneo para obras. }\end{array}$} \\
\hline & & $\begin{array}{l}\text { Presupuesto para capacitación } \\
\text { al personal militar en áreas no } \\
\text { tradicionales. }\end{array}$ & & \\
\hline
\end{tabular}

Fuente: elaboración propia a partir de los resultados obtenidos en la investigación y presentados en la tesis. 
Las misiones de ayuda para la reconstrucción en Cuba, Haití y San Vicente y Granadinas implicaron para el Estado un gasto de \$53.145.562,62 USD, que, si bien pudieron ser utilizados para obras dentro del Ecuador, fueron utilizados en países que atravesaron situaciones adversas. Esta inversión internacional se realizó sobre la base de las decisiones racionales del Ecuador en el marco de su política exterior de impulsar la inserción estratégica en el plano internacional, y lograr visibilidad de un Estado pequeño a través de una de sus instituciones más sólidas, las Fuerzas Armadas.

\section{Conclusiones}

En atención a la pregunta planteada y sobre la base de la teoría utilizada para explicar la vinculación de las Fuerzas Armadas en situaciones de emergencia en desastres naturales a partir de la experiencia ecuatoriana en las misiones de ayuda para la reconstrucción en Cuba, Haití y San Vicente y Granadinas, se llegó a las siguientes conclusiones:

a. Por su naturaleza, las Fuerzas Armadas no son una institución humanitaria. Su razón de ser es proteger al Estado de las agresiones externas que amenacen el territorio y la soberanía. Esta protección encomendada al Ejército tiene directa relación con la necesidad del Estado de garantizar su existencia en el sistema internacional.

b. Las Fuerzas Armadas, como se las conoce en Ecuador, y los ejércitos en general, representan al Estado y actúan sobre la base de las órdenes dadas por las autoridades estatales. Esta vinculación de las entidades encargadas de la seguridad con las autoridades políticas que toman las decisiones, vuelve legítimas y legales las actividades de agresión y/o defensa, dependiendo de la definición de política exterior del Estado. En el caso ecuatoriano, las Fuerzas Armadas son defensivas.

c. El Estado, conforme se indicó a partir de la teoría realista, es racional y unitario. Aplicando esta idea al caso de estudio, se desprende que el Ecuador es un Estado racional que aplica una política exterior encaminada a proteger su existencia en el sistema internacional. Esta necesidad de existencia en un sistema anárquico ha llevado a que la generalidad de la política exterior ecuatoriana se centre en la defensa del territorio nacional y de la soberanía. Para ello, las Fuerzas Armadas se han preocupado tradicionalmente por la defensa nacional, la protección del territorio, tanto continental como insular, aéreo y mar territorial, y de la soberanía. Así mismo, la vinculación política de las Fuerzas Armadas, al haber nacido al mismo tiempo de la creación del Estado, se ha evidenciado en varias épocas de la República, no solo en aspectos militares, sino también de desarrollo, como ocurrió en la década de los 70 .

d. De acuerdo con la visión realista de Estados pequeños, estos se esfuerzan por no desaparecer del sistema 
internacional. En este sentido, sus decisiones racionales se toman en virtud del conocimiento del tamaño y de las capacidades materiales que les permitan ganar poder en el sistema o, al menos, mantener el poder que ya tienen. En este sentido, Ecuador no amenaza la existencia de sus vecinos, es un Estado territorialmente pequeño en comparación con Perú y Colombia; tiene una economía dependiente de las exportaciones de materias primas y ha mantenido una política inestable con cambios de gobierno y de normativa constitucional permanentes. Sin embargo, las Fuerzas Armadas son una de las instituciones más sólidas y organizadas, con un estatus especial en la vida nacional, puesto que no solo tienen mérito por haber defendido el territorio ecuatoriano, sino también por haberse vinculado con el desarrollo y con la defensa de la democracia.

e. De acuerdo con la Constitución de la República del Ecuador, las Fuerzas Armadas son una institución protectora de los derechos, libertades y garantías de los ciudadanos. Su misión tradicional es la protección y defensa de la integridad territorial y de la soberanía, y, de acuerdo con la legislación, hasta agosto de 2018 tuvieron la misión complementaria de apoyar en la seguridad integral. Este contexto constitucional, junto con los objetivos, políticas y metas del Plan Nacional de Desarrollo y del Plan Nacional de Seguridad Integral permitieron que las Fuerzas Armadas se involucraran en activi- dades adicionales a sus actividades tradicionales. Entre estas actividades se encuentran la lucha contra el narcotráfico y la gestión de riesgos.

f. La gestión de riesgos, especialmente en el caso de los desastres naturales, demandan la atención integral del Estado para hacer frente a las emergencias de manera rápida y eficiente. Hay que considerar que, en el ámbito internacional, la Organización de Naciones Unidas se ha pronunciado sobre la responsabilidad primigenia del Estado para responder de manera diligente ante situaciones de emergencia por desastres naturales. De hecho, la respuesta internacional en situaciones de emergencia no ha sido normada, como ocurre con los casos de conflictos armados, lo que ha llevado a que la ayuda en desastres sea vista como la aplicación de intereses bajo la justificación de la solidaridad internacional y la expresión de los más nobles sentimientos humanos de ayuda y protección a la vida e integridad de las personas.

g. En el caso específico del Ecuador, el empleo de militares en actividades de emergencia por desastres naturales respondió a dos visiones específicas: a) aplicación utilitaria de las Fuerzas Armadas a través del Cuerpo de Ingenieros del Ejército, y b) necesidad racional de posicionamiento y reconocimiento internacional bajo un contexto de solidaridad internacional y cooperación encaminada a la inserción estratégica del Estado ecuatoriano en la región. Esta aplicación se reali- 
zó bajo tres conceptos importantes: a) seguridad integral, que incluye tanto a la defensa nacional como a la gestión de riesgos; b) determinación de las Fuerzas Armadas como institución protectora de derechos; c) aplicación de política exterior de respeto a la igualdad jurídica de los Estados y no injerencia en decisiones estatales.

h. La utilización de militares en misiones de ayuda para la reconstrucción respondió a una idea práctica de experiencia y organización de esta institución armada. Sin embargo, considerando que estas misiones se dieron bajo acuerdos bilaterales directos, era necesario que el Estado ecuatoriano capacitara al personal de las misiones, de manera que su actuación no contradijera las buenas intenciones estatales, ni afectara los derechos humanos, puesto que actividades contrarias a estas visiones hubieran producido el efecto contrario a los objetivos de prestigio y reconocimiento internacional. Así también, el respeto a los derechos humanos y a la autonomía del Estado beneficiado permitió que los militares ecuatorianos no fueran considerados un peligro, ni que el Estado fuera visto como intervencionista en la política interna de los Estados beneficiados. A partir de lo mencionado, se desprende que las Fuerzas Armadas no son una institución humanitaria como tal; sin embargo, la preparación en derechos humanos y la reformulación de intereses estatales pueden convertirlas en importantes colaboradores para misiones alternativas a la protección del territorio, la soberanía y la defensa nacional. Esta vinculación con los derechos humanos en un contexto internacional, y la capacidad de respuesta inmediata y eficiencia, permite que el Estado racional utilice a su ejército en actividades que le permitan conseguir sus objetivos estratégicos, a partir de una política exterior racional encaminada a intereses de poder y seguridad, que garanticen la existencia del Estado en un contexto internacional anárquico.

\section{Referencias}

Asamblea Nacional. (1906). Constitución Política de la República del Ecuador. Quito - Ecuador: Registro Oficial $n^{\circ} 262$.

Asamblea Nacional Constituyente. (1945). Constitución Política del Ecuador. Registro Oficial $n^{\circ} 228$.

Asamblea Nacional Constituyente. (1967). Constitución de la República del Ecuador. Registro Oficial $n^{\circ} 113$.

Asamblea Nacional Constituyente. (1998). Constitución Política de la República del Ecuador. Riobamba Ecuador: Registro Oficial $n^{\circ} 1$.

Asamblea Nacional Constituyente. (2008). Constitución de la República del Ecuador. Quito: Registro Oficial $n^{\circ} 449$.

Beach, D. (2012). Analyzing Foreing Policy. New York: Palgrave Macmillan.

Bonilla, A. (1997, abril). A propósito del diálogo Ecuador-Perú Límites y 
horizontes de la negociación. ICOnos. Revista de Ciencias Sociales, 1, 68-75.

Bonilla, A. (2006). Política exterior del Ecuador: 25 años de vulnerabilidad. Revista de la Asociación de Funcionarios y Empleados del Servicio Exterior Ecuatoriano, 44, 165181.

Carranza B., J. A. (2002). Relaciones bilaterales Ecuador y Perú: una propuesta de agenda de política exterior. En A. Bonilla (ed.), Orfeo en el infierno una agenda de política exterior ecuatoriana (pp. 137-229). Quito - Ecuador: Flacso, sede Ecuador.

Congreso Constitucional. (1835). Constitución de la República del Ecuador. Ambato.

Congreso Constituyente. (1830). Constitución del Estado del Ecuador. Riobamba - Ecuador.

David, C. P. (2008). La guerra y la paz. Enfoques contemporáneos sobre seguridad y estrategia (Covadonga Morales Bertrand). Barcelona: Icaria \& Antrazyt editorial S.A.

De la Torre Arauz, P. (2013). Los constructores del Estado Nacional 18302010 en el cerebro político del Ecuador $\left(1^{\circ}\right.$ ed). Quito - Ecuador: Senplades.

Fuentes Julio, C. (2012). Seguridad humana: referencias conceptuales y enfoque práctico para América Latina. En F. Rojas Aravena (ed.), Seguridad humana: nuevos enfoques ( $1^{\circ}$ ed., pp. 33-54). San José C.R.: Flacso.
Goetschel, L. (1998). The Foreing and Security Policy Interests of Small States in Today's Europe. En L. Goetschel (Ed.), Small States Inside and Outside the European Union: Interests and Policies (pp. 13-31). Boston, MA: Springer Science+Business Media Dordrechl.

Grosso, E. C. (2012). La impronta legal de la participación de las Fuerzas Armadas en la seguridad pública : lucha contra el narcotráfico en América del Sur. Urvio - Revista Latinoamericana de Estudios de Seguridad, (12), 97-109. https://doi. org/10.17141/urvio.12.2012.1170

Hey, J. A. K. (2003). Introducing Small State Foreing Policy. En J. A. K. Hey (Ed.), Small States in World Politics. Explaining Foreing Policy Behavior (pp. 1-11). Bouder, Colorado: Lynne Rienner Publishers.

Karns, M., \& Mingst, K. A. (2004). "International Organizations". The Politics and Processes of Global Governance. Boulder y Londres: Lynne R ienner Publishers, Inc.

Macías Núñez, E. (2010). El floreanismo. En D. Andrade Aguirre (Ed.), Historia militar del Ecuador (pp. 245-292). Quito - Ecuador: Ministerio de Defensa Nacional.

Miduvi. (2013). Convenio Interinstitucional entre el Ministerio de Desarrollo Urbano y Vivienda del Ecuador y el Ministerio de la Construcción de Cuba para la Cooperación en el Sector de la Construcción. Registro Oficial $n^{\circ} 56$. 
Ministerio Coordinador de Seguridad. (2014). Plan Nacional de Seguridad Integral. Quito - Ecuador: Senplades.

Ministerio de Relaciones Exteriores. (1995). El problema territorial ecuatoriano-peruano del conflicto del Cenepa a la búsqueda de la paz. Quito - Ecuador: Ministerio de Relaciones Exteriores de la República del Ecuador.

Moncayo, P. (1994). Ecuador: geopolítica, poder y seguridad. Sangolquí - Ecuador: Editorial Escuela Politécnica del Ejército.

Morgenthau, H. J. (1986). Política entre las naciones. La lucha por el poder y la paz. (K. W. Thompson, Ed., H. W. Olivera, Trad.) ( $1^{\circ}$ ed.). Buenos Aires: Grupo Editor Latinoamericano GEL.

Navarrete, C. (6 de Junio de 2018). Misiones de ayuda para la reconstrucción: directrices generales y Marhec.

Oxfam (2011). ¿De quién es esta ayuda? Ayuda politizada en crisis y conflictos. Reino Unido: Oxfam Internacional. Recuperado de https://www.oxfam. org/sites/www.oxfam.org/files/file_ attachments/bp145-whose-aid-anyway-100211-es_0_4.pdf

Peña Cobeña, J. (25 de mayo de 2018). Misiones de ayuda humanitaria para la reconstrucción en el Caribe.

Pérez, D. (12 de marzo de 2016). Las Fuerzas Armadas ecuatorianas son un reflejo de la sociedad. Diario EI Telégrafo. Recuperado de https:// www.eltelegrafo.com.ec/noticias/ politica/3/las-fuerzas-armadasecuatorianas-son-un-reflejo-de-lasociedad

Pérez de Armiño, K. (2006). El concepto $y$ el uso de la seguridad humana: análisis crítico de sus potencialidades y riesgos. Revista Ciddob d'Afers Internacionals, (76), 59-77.

Rojas Aravena, F., y Álvarez Marín, A. (2012). Seguridad humana. Un estado del arte. En F. Rojas Aravena (ed.), Seguridad Humana: Nuevos Enfoques ( $1^{\circ}$ ed., pp. 9-32). San José C.R.: Flacso.

Salgado Espinoza, R. (2017). Small Builds Big. How Ecuador and Uruguay Contributed to the Construction of Unasur. Quito: Flacso Ecuador.

Senplades. (2009). Plan Nacional para el Buen Vivir 2009-2013. Construyendo un Estado plurinacional e intercultural. Quito - Ecuador: Senplades. Recuperado de http://www. planificacion.gob.ec/wp-content/ uploads/downloads/2012/07/Plan_ Nacional_para_el_Buen_Vivir_(version_resumida_en_espanol).pdf

Trujillo Vásquez, J. C. (2006). Teoría del Estado en el Ecuador: estudio de Derecho Constitucional $\left(2^{\circ}\right.$ ed., vol. 8). Quito: Corporación Editora Nacional.

Velásquez Flores, R., y González Cruz, S. G. (2014). Realismo clásico. En J. A. Schiavon Uriegas, A. S. Ortega Ramírez, M. López-Vallejo Olvera y R. Velásquez Flores (eds.), Teorías de las relaciones internacionales en el siglo XXI: Interpretaciones críticas desde México (pp. 211-226). Pue- 
bla: Benemérita Universidad Autónoma de Puebla, El Colegio de San Luis, A.C., Universidad Autónoma de Baja California, Universidad Autónoma de Nuevo León, Universidad Autónoma del Estado de Puebla.

Vivas, P. (2015). Un análisis del empleo de las Fuerzas Armadas en emergencias y catástrofes. El Modelo Español. En S. Alda Mejías y S. De Sousa Ferreira (Eds.), La multi- dimensionalidad de la seguridad nacional: retos y desafíos de la región para su implementación (pp. 361388). Madrid: Instituto Universitario General Gutiérrez Mellado.

Wohlforth, W. C. (2012). Realism and Foreign Policy. En S. Smith, A. Hadfield y T. Dunne (Eds.), Foreign Policy. Theories, Actors, Cases (second edition, pp. 35-53). United Kingdom: Oxford University Press. 
